\title{
Successful Pregnancy Following Repair of Vesicouterine Fistula Following Repeat Caesarean Section: A Case Report
}

\author{
Dilruba Akter ${ }^{1}$, Salahuddin Shah ${ }^{2}$, Ferdousi Islam ${ }^{3}$, Saria Tasnim $^{4}$ \\ ${ }^{1}$ Assistant Professor (Obs and Gynae), Institute of Child and Mother Health, Matuail, Dhaka 1362, ${ }^{2}$ Assistant Professor, Haematology \\ Department, BSMMU, Dhaka, ${ }^{3}$ Professor and Head of the Department (Obs and Gynae), ${ }^{4}$ Associate professor (Obs and Gynae), Institute \\ Of Child and Mother Health, Matuail, Dhaka 1362
}

\begin{abstract}
:
A lady, para 2, both delivered by caesarean section for prolonged labour, presented with the complaints of menouria and infertility for 3 years following her last caesarean section. She had history of haematuria during menstruation and her menstrual flow is scanty. Her first baby was female and alive, but her second baby was stillborn following prolonged and obstructed labour. This has been continuously blamed by family and others for happening that events. Her fistula was confirmed by ultrasound scan, histerography and cystoscopy. The vesicouterine fistula was treated by local repair with omental patch through transperitoneal approach. Pregnancy following one year of successful repair has turned her from miserable to happiness in life.
\end{abstract}

Key words: Vesicouterine fistula, menouria, haematuria.

[BSMMU J 2012; 5(1):76-78]

\section{Introduction:}

Uterovesical fistula is an abnormal communication between uterine cavity and urinary bladder. It is a rare type of fistula accounting for only $1-4 \%$ of all cases of urogenital fistula. ${ }^{1,2}$ However the incidence of uterovesical fistula has been rising due to increasing incidence of lower segment caesarean section.Uterovesical fistula is seen more after repeat caesarean section rather than after primary. ${ }^{3,4}$ There are few case reports about this condition following vaginal birth after caesarean (VBAC). ${ }^{2}$

There are newer causes for this fistula such as uterine artery embolisation. ${ }^{5}$ The development of fistula is believed to relate to higher attachment of the bladder relating to lower uterine segment, usually secondary to scarring from previous surgery.With an unrecognized bladder injury or suture transfixation of the bladder, a tract may develop between bladder and uterine wall. Jazwik and Jozwik ${ }^{12}$ have proposed a classication of uterovesical fistula which is based on route of menstrual flow. Type 1 (Youssef syndrome) is menouria, amenorrhoea and continence of urine. Type 2 is dual direction menstrual flow via bladder and vagina. ${ }^{12}$ Management of uterovesical fistula can be done either conservative or surgical.Conservative treatment is indicated when the fistula is diagnosed early. Spontaneous healing is reported in $5 \%$ of women. ${ }^{13}$ Surgical treatment is indicated when conservative

Address of Correspondence: Dr. Mossammat Dilruba Akter, Assistant Professor (Obs and Gynae), Institute Of Child and Mother Health, Matuail,Dhaka 1362, Email: dilruba042@yahoo.co.in treatment has failed or in cases involving a large fistula. This surgical treatment can be carried out either by laparoscopy or laparotomy immediately after the diagnosis (in 48 hours) or 3-4 months after diagnosis. The pregnancy rate after repair has been reported to be $31.25 \%-37.55 \%$ with a rate of live birth deliveries of $25 \% .{ }^{13}$

\section{Case Report:}

A lady, para 2, both delivered by caesarean section for prolonged labour, had history of haematuria during menstrual periods following her last caesarean section performed 5 years earlier. She had history of menouria or cyclical haematuria but no urinary incontinence. She had also complains of failure to conceive for 3 years. Her first baby is female and alive and second baby was stillborn and which was a male child. She was in extreme mental agony during her post partum period following her last caesarean section. Her mother in-law and her husband blamed her for loosing the male baby and was neglected by the family members. She noticed menouria when she resumed her cycles 2 months after the LSCS. Her menstrual flow through the vagina was scanty. After that, she was trying for conception but failed to conceive. When her life became unbearable because of torture of her in-laws and husband then she came to Institute of Child and Mother Health for the treatment of menouria and infertility. Her general and systemic examinations were normal. She had a midline vertical sub-umbilical caesarean scar. Pelvic examination revealed a normal sized anteverted uterus and fornices were free. Her fistula was confirmed by ultrasound 
scan (fig-1), hysterography showed the flow of contrast from the uterus to the bladder (fig-2). Cystoscopy showed a fistulous opening in the bladder measuring about $7 \mathrm{~mm}$ in size. It was supratrigonal in position. Intravenous pyelography showed a normal upper renal tract. The bladder capacity was normal. The vesicouterine fistula was then treated by local repair through transperitoneal route. Intraoperatively dense fibrous adhesions were found between the lower segment of the uterus and the

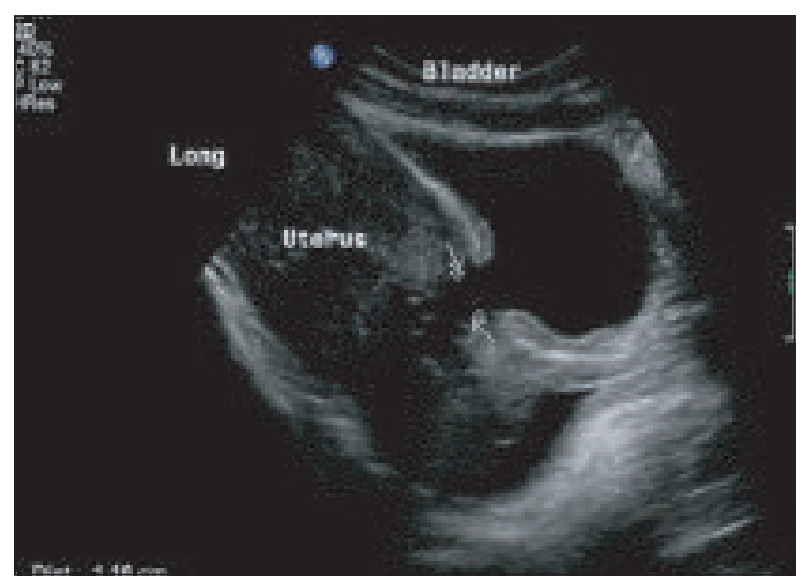

Fig.-1: Uterovesical fistula (arrows) on ultrasound scan

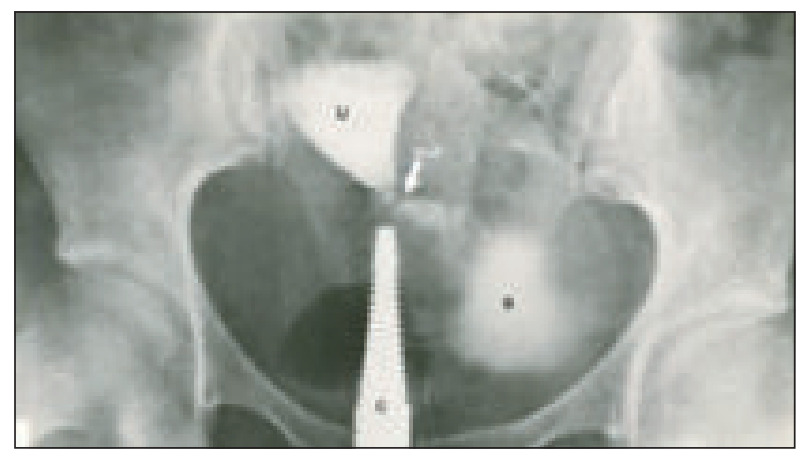

Fig.-2: Hysterography reveals the fistula with dye leaking into the bladder from the uterus

bladder. A 2-3 cm fistula was located in the lower segment scar area. The trigone and ureters were not involved. Separation of bladder from the uterus was very difficult. After separation uterus and bladder wall was repaired in two layers by vicryl 3-0 and uterine wall was repaired by vicryl 1-0. Omental patch was given between uterus and bladder. Continuous bladder drainage was maintained using a Foley's catheter for two weeks. She was then put on injection depot medroxyprogesterone for 3 months to suppress menstruation. After operation her husband left her with the apprehension that his wife had lost everything and will never conceive again. The lady went back to her father's house after operation. Her life became miserable because she was living alone despite of having her daughter and husband. When the patient resumed her cycles four months later she did not have menouria and her menstrual flow was normal. One day, during her follow up she told me, "How can I alive without my only child?" Her mother in- law did not allow her to communicate with her daughter. By this time her husband was getting ready for second marriage. But the marriage was stopped with the help of law enforcing authority. For the last six months husband used to come to his wife and they had coitus at every 15 days. Two months ago, suddenly she felt nausea and vomiting and for that reason she got done urine for pregnancy test and result was positive. This information was communicated to her husband and mother in- law. Being confirmed of her recent pregnancy her husband brought her to his house. Now she is happy in living with her husband and daughter. Her husbands as well as her mother in-law take care of her and ensuring her diet, rest and medical check up.

\section{Discussion:}

Caesarean section is the most common cause for uterovesical fistula. . It is a rare type of fistula accounting for only $1-4 \%$ of all cases of urogenital fistula. ${ }^{1,2}$ It is interesting to note that prior to 1947 operative vaginal delivery was the commonest cause of vesicouterine fistula and total urinary incontinence was the usual complaint. However in post caesarean vesicouterine fistula menouria is an important symptom while incontinence may or may not be there. ${ }^{6}$ As the bladder injury is more during repeat caesarean, the incidence rises with the number of caesarean sections. Jozwic et al ${ }^{12}$ also observed that vesicouterine fistula following caesarean section were more common on the left side of the uterus due to dextrorotation. ${ }^{3}$ It can occur after forceps/vacuum delivery or after vaginal birth following previous caesarean section.

The classical Youssef's syndrome is characterized by menouria, absence of urinary incontinence, vesicouterine fistula and amenorrhoea despite a patent cervical canal.This is explained by the differential pressure gradient between the uterus and the bladder and the sphincter action of the isthmus, which facilitates passage of blood from the uterus into the bladder. ${ }^{7}$

Our case is not a classical vesical Youssef's syndrome as she was not amenorrhoeic. She had hypomenorrhoea. Absence of leakage of urine is due to high level of the fistula in the bladder. To demonstrate the fistula 
the hysterogram should be ideally performed with a short tipped canula. ${ }^{6}$

Endometriosis of the bladder is a differential diagnosis for menouria. Here, along with other symptoms of endometriosis, menstrual flow occurs through the vagina in addition to menouria. Imaging studies help in outlying the fistulous tract and planning treatment.In patients with menouria, hysterography reveals the fistula with dye leaking into the bladder from the uterus.However, in recent times helical CT with sagittal reformation performed soon after hysterography helps one to visualize the fistulous tract clearly. ${ }^{7}$

Vesicouterine fistula following caesarean section may heal spontaneously

with involution of the puerperal uterus. Spontaneous healing may occur in $5 \%$ of cases. ${ }^{1}$ When it does not, continuous hormonal therapy can be given to suppress menstruation for 3-6 months as first line of therapy. Suppression of menstruation can be tried with progesterone or Latinizing hormone. In our case injectable progesterone was given to suppress menstruation. There are a few other case reports of the simple technique of cystoscopic fulguration as a primary approach with successful outcome. ${ }^{8}$ Laparoscopic repair of VUF is now evolving as a new procedure with minimal blood loss and less morbidity. ${ }^{9}$ The other approach is to separate the bladder from uterine wall, excise the fistula and close the bladder and uterus separately. The bladder should be closed in two layers without tension. To strengthen the repair myouterine flap and omentum has been interposed though the risk of postoperative bowel obstruction is present in the later as reported by Char Et al. ${ }^{10}$ Successful pregnancy and delivery by caesarean section after fistula repair has been reported. ${ }^{11}$ In our case successful repair of the uterovesical fistula was done and patient became pregnant This pregnancy has produced a new turn of life from misery to happiness in this lady.

\section{Conclusion:}

Vesico-uterine fistula can still be encountered especially with the wide-spread use of lower segment caesarean section and should be suspected when multiparous women with history of repeated caesarean sections presents with menouria. Meticulous practice of obstetric and surgical principles during caesarean section can prevent the women from this type of miserable morbidity.

\section{References:}

1. Kilinc F,Bagis $\mathrm{T}$.Unusual case of post-caesarean vesicouterine fistula (Youssef's syndrome ) International journal of Urology. 2003; 10(4): 235-8.

2. Park QR, Kim TS. Sonographic diagnosis of vesicouterine fistula .Ultrasound in obstetric and gynaecology. 2003; 22(1): $82-4$.

3. Jozwik M,Jozwick M, Lotocki-W, Vesicouterine fistula -an analysis of 24 cases from Poland -Int J Gynecol Obstet, 1997; 5792): 169-72.

4. hadzi-Djokie jB, Pejcic TP, Colovic VC, Vesicouterine fistula :report of 14 cases BJU Int 2007; 100(6): 1361-3.

5. Sultana C J, Aizenman. L, Chon JK- Vesicouterine fistula; after uterine artery embolisation: a case report, Am J obstet Gynecol 2002; 187: 1726-27.

6. Leon-Tracer M: Vesicouterine Fistula-A review, Obstet \& Gynecol Surv 1986; 41(12): 743-753.

7. Smayra T, Ghossain MA, Buy JN, Moukarzel M, Jacob D, True JB. Vesicouterine Fistulas:Imaging findings in three cases, AJR Am J Roentgenol 2005; 184(1): 134-142.

8. Tarhan F, Erbay E, Penbequl N, Kuyumcuo U, Minimal invasive treatment of vesicouterine fistula; a case report- Int Urol 2007; 3993): 791-3.

9. Das Mahapatra P, Bhattacharyya P. Laparoscopic intraperitoneal repair of high-up urinary bladder fistula: a review of 12 cases Int Urogynecol J 2007; 18(6): 635-9

10. Char D, Krasnokutsky S, Frischer Z, Shah SM, Bayshtok J, Khan SA. Surgically correcting a vesicouterine fistula with a myouterine flap. A case report. J Reprod Med 1997; 42(6):3724.

11. Issa MM, Schmid HP, Stamey TA. Youssef'ssyndrome: Prevention of Uterine function with subsequent successful pregnancy following surgical repair. Uro Int 1994; 52(4): 220-2.

12. Jozwik M, Jozwik M. Clinical classification of vesicouterine fistula. Int J Gynecol 2000; 70(30):353-7.

13. Porcaro AB, Zicari M Vesicouterine fistula following caesarean section.International Journal of Urology \& Nephrology ;2003: $335-44$. 